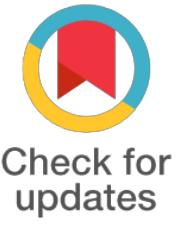

${ }^{*}$ For correspondence:

trinhnhuthuy@gmail.com

Competing interests: The authors declare that no competing interests exist.

Received: 2017-05-15

Accepted: 2017-07-03

Published: 2017-09-05

Copyright The Author(s) 2017. This article is published with open access by BioMedPress (BMP).

This article is distributed under the terms of the Creative Commons Attribution License (CC-BY 4.0) which permits any use, distribution, and reproduction in any medium, provided the original author(s) and the source are credited.

\section{Functional analysis of human type 2 diabetic adipose tissue-derived mesenchymal stem cells}

Nhu Thuy Trinh, Toshiharu Yamashita, Kinuko Ohneda, Kenichi Kimura, Georgina To'a Salazar, Fujio Sato, Osamu Ohneda

2374 National Highway

1 Trung My Tay Ward, District 12, Ho Chi Minh City 707800, Vietnam

\section{Abstract}

Background: Stem cell therapy has recently shown promise in the prevention of diabetic complications due to its regenerative potential. The possible applications of human diabetic adipose tissue-derived mesenchymal stem cells (dAT-MSCs) in cell therapy are limited because their characteristics are still not fully understood. Aims: This study aimed to characterize dAT-MSCs in vitro and to investigate the potential application of dAT-MSCs in wound healing. Materials and Methods: dAT-MSCs were characterized under normoxic and hypoxic conditions in vitro and evaluated wound healing capacity in the ischemic flap mouse model. Results: Early growth response factor-1 (EGR-1) and its target genes were highly expressed in dAT-MSCs in comparison to nAT-MSCs, resulting in increasing of genes and protein associated with cell adhesion, insulin resistance, and impaired wound healing. Interestingly, under hypoxic conditions, hypoxia-inducible factor-1a (HIF-1a) can bind to the EGR-1 promoter in dAT-MSCs, but not in nAT-MSCs. The effects of EGR-1 were inhibited by shEGR-1 and PD98059. Mice injected with shEGR-1- dAT-MSCs were improved their wound healing capacity. Furthermore, we found that human nAT-MSC-derived microvesicles (nMVs) could improve dAT-MSC function by altering miRNA and mRNA expressions, which enhanced their migration ability in vitro and wound healing capacity in the ischemic flap mouse model. Conclusion: Our study suggests that dAT-MSCs may contribute to delay wound healing. Interrupting the expression of EGR-1 in dAT-MSCs or transfecting nMVs to dAT-MSCs may be a useful treatment for chronic wounds in diabetic patients.

\section{Keywords}

stem cells, diabetes, EGR-1, microvesicles

\section{Funding}

\section{References}

